\title{
A Phenomenology of Pain - Eine Phänomenologie des Schmerzes
}

$\begin{aligned} \text { Authors: } & \text { Claudia Bozzaro } \\ \text { Submitted: } & \text { 10. April } 2018 \\ \text { Published: } & 16 . \text { May } 2018 \\ \text { Volume: } & 5 \\ \text { Issue: } & 4 \\ \text { Affiliation: } & \text { Institut für Ethik und Geschichte der Medizin. Freiburg, } \\ & \text { Deutschland } \\ \text { Languages: } & \text { German } \\ \text { Keywords: } & \text { Pain, Body-experience; Time-experience } \\ \text { DOI: } & 10.17160 / \text { josha.5.4.415 }\end{aligned}$

Journal of Science, Humanities and Arts 


\title{
Eine Phänomenologie des Schmerzes / A Phenomenology of Pain1
}

Dr. Claudia Bozzaro

Institut für Ethik und Geschichte der Medizin

Stefanmeierstr. 26

79104 Freiburg

E-Mail: bozzaro@egm.uni-freiburg.de

Phone (+) 49-(0)761-2035040

\section{Zusammenfassung}

Dieser Beitrag skizziert eine Phänomenologie des Schmerzes im Hinblick auf vier Dimensionen menschlicher Existenz: Leiblichkeit, Zeitlichkeit, Intersubjektivität und Selbsterleben. Das Erleben physischer Schmerzen aus der Innenperspektive des Leidenden soll dabei möglichst präzise und umfassend zur Sprache gebracht werden. Im Text wird daher nicht in erster Linie auf wissenschaftliche Analysen des Schmerzes Bezug genommen, es werden vielmehr literarische Schmerzdarstellungen und Erzählungen von Schmerzpatienten zugrunde gelegt.

\begin{abstract}
This article draws a phenomenology of pain focusing on four aspects of human existence: bodyexperience, time-experience, intersubjectivity and self-experience. The pain-experience will be presented from the perspective of the person who suffers. For this the article takes into account not only scientific descriptions of pain but also fictional description in literature and reports from pain-patients.
\end{abstract}

Schlüsselwörter: Schmerz, Leiblichkeit, Zeitlichkeit.

Keywords: Pain, Body-experience; Time-experience

\section{Einleitung}

Das Thema Schmerz hat aktuell Hochkonjunktur. In Anbetracht der ständig wachsenden Zahl chronischer Schmerzpatienten, aber auch onkologischer Patienten und hochbetagter Menschen ist dies nicht verwunderlich. In den meisten Beiträgen zum Thema Schmerz werden Zahlen über und rund um den Schmerz vorgestellt und besprochen, es wird darübergeschrieben, wie Schmerzen - aus medizinischer, psychosomatischer, psychologischer usw. Perspektive - zu verstehen sind, welche Funktion, Bedeutung oder welchen Sinn sie haben könnten. Nicht zuletzt werden Umgangs- und Bewältigungsstrategien vorgestellt. Doch nur selten wird einfach einmal dargestellt, wie Schmerzen sind: Wie ist es, Schmerzen zu haben, wie fühlen sie sich an, wie werden Schmerzen erlebt?

\footnotetext{
${ }^{1}$ Bei diesem Text handelt es sich um einen Abdruck eines bereits veröffentlichen Textes, der unter diesem Titel in der Zeitschrift Imago Homini 22(2)2015 erschienen ist.
} 
Als Antwort auf diese Fragen soll in diesem Beitrag eine kleine Phänomenologie, eine einfühlend-deskriptive Darstellung des Schmerzerlebens, versucht werden. Dabei wird das Schmerzerleben in Bezug auf vier unterschiedliche Aspekte angegangen: Leiblichkeit, Zeitlichkeit, Intersubjektivität und Selbsterleben. Es geht um die Schilderung dessen, was im Schmerz passiert, was der Schmerz bewirkt - und zwar aus der Innenperspektive. Dabei steht unweigerlich die Person im Zentrum, die den Schmerz erleidet. Über Schmerzen als abstrakte Entität zu sprechen, macht nur bedingt Sinn und kann unter Umständen sogar gefährlich werden. Schmerzen gibt es nicht losgelöst von einem Lebewesen, Schmerzen werden erlebt und erfahren. Natürlich ist es möglich, in einem übertragenen oder metaphorischen Sinn auch von einem „Weltschmerz" zu sprechen. Aber dabei handelt es sich eben um eine Übertragung, die im Folgenden nicht berücksichtigt werden soll. Wer über den Schmerz etwas aussagen will, muss stets vor Augen haben, dass Schmerzen immer die individuellen, besonderen Schmerzen einer bestimmten Person sind. Um dem Rechnung zu tragen, werden sich die folgenden Ausführungen nicht nur auf theoretische, wissenschaftlich fundierte Beschreibungen des Schmerzes stützen, sondern ebenso auf literarische Darstellungen des Schmerzerlebnisses und nicht zuletzt auch direkte Aussagen von chronischen Schmerzpatienten einbeziehen. Diese Zeugnisse werden meinen Text immer wieder auf- und unterbrechen. Sie dienen nicht nur der Selbstvergewisserung und Untermauerung des Gesagten. Vielmehr gewähren sie unmittelbare Einblicke in eine Realität, die sich ein relativ gesunder, weitgehend schmerzfreier Mensch nur schwer vorstellen kann und die letztlich keine Theorie gebührend zu erfassen vermag. Darunter sind Dokumente des persönlich Erlebten, die chronische Schmerzpatienten dankenswerterweise zur Verfügung gestellt haben. $^{2}$

\section{Der Leib im Schmerz}

\section{Der kurze Schmerz}

Ein ungestörtes Verhältnis zum eigenen Leib zeichnet sich durch die Verborgenheit desselben aus. Der Leib wird wird zwar ständig beansprucht, mal in passiver Weise, wie z.B. im Schlaf, mal aktiv, wie beim Sport, er wird eingesetzt, um Handlungen auszuführen, um mit anderen Menschen zu kommunizieren und um mit der Umwelt zu interagieren. Doch dabei wird der eigene Leib oder einzelne seiner Teile nicht bewusst wahrgenommen, er bleibt in

\footnotetext{
${ }^{2} \mathrm{Vgl}$. www.krankheitserfahrungen.de (zuletzt abgerufen am 21.12.2014).
} 
eigentümlicher Weise verborgen. Aus dieser Verborgenheit wird er durch den plötzlichen Schmerz herausgerissen.

Der größte Teil des Körpers spricht nur, um zu leiden. (Paul Valéry, Cahiers/Hefte)

Der plötzliche Schmerz durchzieht den Körper und reißt ihn dadurch aus seiner Unmittelbarkeit heraus. Reflexartige, nicht intensionale Bewegungen, ein unkontrollierter Schrei brechen aus dem von Schmerzbetroffenen heraus. Es sind meistens Reflexe, die Flucht, Rückzug und Ablehnung zu signalisieren scheint. Nach dem Einbruch des Plötzlichen, nach dem ersten Schock, lässt der erste akute Schmerz langsam nach. Es tritt nun ein zweites Moment der Schmerzerfahrung ein: das Verletztsein. Der akute Schmerz wandelt sich in einen diffuseren und anhaltenden, Schmerz, der die Information im Körper weiterträgt: Ich bin verletzt. Der Schmerz ist nun zu einem Zustand geworden, der im eigenen Leib abläuft und von dem sich der vom schmerz Betroffene sich deshalb nicht distanzieren kann. Die eigene Leibwahrnehmung verändert sich grundlegend. Die Aufmerksamkeit ist auf die schmerzende Körperpartie gerichtet, das unmittelbare Aufgehen in der Umwelt, in Tätigkeiten ist nicht mehr möglich. In extremeren Fällen drängt sich die schmerzende Körperpartie wie ein fremder Eindringling ins Bewusstsein und erinnert penetrant daran, dass es ein Teil von Einem ist, dass man Rücksicht auf es nehmen muss, sonst wird er sich sogleich mit dem nächsten schmerzvollen Stich rächen. Das schmerzende körperteil wird als fremdes Objekt wahrgenommen, als etwas was in einem ist obgleich man es gerne wegstoßen würden.

...es ist da etwas, das ist von mir und doch nicht von mir - anders als ich in mir. Unabweisbar wie ich. Und eine Art anderes Ich, welches das Ich bekriegen will-ein Empfundenes, das alles andere zu vernichten trachtet... (Paul Valéry, Cahiers/Hefte)

Der Schmerz wird als etwas empfunden, das nicht sein soll, das vergehen soll, weil er weh tut. Doch ein Rückzug von ihm ist nicht möglich, denn der Schmerz ist Teil des eigenen Körpers, der eigenen Leiblichkeit und somit Teil von einem selbst geworden. ${ }^{3}$

Die Erfahrung eines unerwarteten, plötzlichen Schmerzes, der unmittelbar die Leibgebundenheit der eigenen Existenz erfahren lässt ist meistens eine Erlebnis von begrenzter Dauer. Ein plötzlicher Schmerz kann zwar einen momentanen Bruch oder eine Störung in Lebensalltag

\footnotetext{
${ }^{3}$ Eine ausführlichere Beschreibung der Leiblichkeitserfahrung im Schmerz findet sich bei Grüny (2004).
} 
herbeiführen, auch eine momentane Veränderung der eigenen Leibwahrnehmung. Doch nach einem meist absehbareren Zeitraum gelangt alles wieder zur Normalität.

\section{Der chronische Schmerz}

Doch was passiert, wenn der Schmerz kein kurzer, vorübergehender Schmerz ist, sondern ein permanenter, einer, der immer wieder kommt? Die Erfahrung von chronischen Schmerzen ist keine Erfahrung, die sich ohne weiteres verallgemeinern lässt wie Erfahrungen von kurzen Schmerzen. Betroffen davon sind allerdings auch nicht wenige Menschen - im Gegenteil: Millionen von chronischen Schmerzpatienten, onkologischen Patienten und älter werdenden Menschen leiden darunter. Sie erleben einen Schmerz, der eine massive Veränderung in allen Bereichen ihrer Existenz bewirkt, angefangen bei der eigenen Leiblichkeit.

... aber was wisst ihr von Schmerzen, was kennt ihr von all den kleinen Tricks, die man ausführen muss und die einem den ganzen Geist in beschlag nehmen, in einer solchen Position zu sitzen, das Bein gerade auf diese Art legen, so die Kleider anziehen, da die Glieder auf genau diese Art ruhen können, all das, kennt ihr die Schlaflosigkeit, kennt ihr den Schlaf, der eigentlich Schlaflosigkeit ist, bloß überdeckt und nie erfrischend, weil der Schmerz wie ein dumpfer cantus firmus nicht von Schlafmitteln gelöscht werden kann... (Peer Hultberg, Requiem.)

Ein lang anhaltender Schmerz an einer beliebigen Körperstelle fördert meistens Fehlhaltungen, Muskelverspannungen, Versteifungen und somit das Aufkommen weiterer „Schmerzherde“. Wenn mal nicht das Bein weh tut, so zieht es im Rücken. Wenn es im Kopf nicht hämmert, dann sticht es im Nacken. Der eigene Körper macht nicht mehr das, was von ihm erwartet wird, was man ihm befiehlt. Im Gegenteil: Er entwickelt ein Eigenleben. Dass man über den eigenen Körper verfügen kann, ist für die meisten Menschen eine Selbstverständlichkeit. Erst kranke, schmerzgeplagte oder alte, gebrechliche Menschen machen die leidvolle Erfahrung, dass sich dieses als normal erlebte Leibverhältnis radikal umkehren kann, dass man selbst zum Sklaven des eigenen Körpers werden kann. ${ }^{4}$ Denn es interessiert den Schmerz nicht, welche Pläne oder Aufgaben, welche Interessen oder Wünsche man hat. Es interessiert ihn genauso wenig, was die eigene Familie, der Vorgesetze und die Kollegen, Freunde und Bekannten denken, meinen, wollen. Wenn er wüten will, dann wütet er.

Patientin: Andrea Müller

Gut, es war dann im Laufe der Jahre einfach so. Es war halt eine deutliche Progredienz und ich musste eigentlich so eins nach dem anderen von den Dingen, die für mich wichtig waren,

\footnotetext{
${ }^{4}$ Zum Zusammenhang von körperlichem Leiden, Zeit und Altern: Bozzaro (2014).
} 
die mir Spaß gemacht haben, ja - die gingen dann nicht mehr. Also Sport musste ich aufhören, Gitarre spielen, Musik machen und diese ganzen Dinge. [...] Was dazu kam, war eben das Berufliche. Ich habe dann nur noch halbtags arbeiten können. Und das war aber noch in Ordnung. Das ging dann auch noch soweit und das war auch wichtig. Und nach zwei Jahren war das aber so, dass der Arbeitgeber mir dann gekündigt hat. Was auch noch einmal ein ziemlicher Einschnitt war.

Der schmerzgeplagte Körper ist längst nicht mehr ein Medium, durch das ein Mensch mit der Welt interagieren kann, Arbeiten verrichten oder an Aktivitäten teilnehmen kann. Es ist nicht mehr der Körper, mit dem man selbstverständlich auf andere Menschen zugeht. Es ist ein geplagter Körper, den man nicht selten vor den Blicken der Anderen verstecken will. Das gelingt jedoch gerade während einer Schmerzattacke nicht. Im Gegenteil, bei einer heftigen Schmerzattacke hat sich der Schmerzleidende nicht mehr unter Kontrolle und nolens volens drängt sein Inneres nach Außen und wird somit für alle sichtbar. Die Intimität wird aufgebrochen. Scham ist ein Gefühl, das Schmerzpatienten gut kennen.

Der Körper des Schmerzleidenden ist ein Körper, der nicht mehr unmittelbar für Eindrücke von Außen offen ist. Wenn der Migräneschmerz akut ist, soll am besten gar nichts die fünf Sinne affizieren. Berührungen - und seien es die eines geliebten Menschen - können unangenehm bis qualvoll sein. Der eigene Körper wird zu einer Mauer, ein Zaun bestückt mit Stacheldraht, eine eisige Wand, die einen einsperrt und von alledem, was bisher das Leben ausgemacht hat, fern hält, entfremdet. Der Schmerz reduziert die Person auf ihren physischen Körper, einen Körper, der als Feind, Gegner, Eindringling erlebt wird.

Wenn nicht nur ein Teil des Körpers schmerzt, sondern der gesamte - wie es bei Fibromyalgiepatienten, aber auch bei älteren Menschen der Fall sein kann - so ist es möglich, dass dieser Entfremdungsprozess zu einer völligen Ablehnung des eigenen Leibes führt, die in ein Ekelgefühl vor sich selbst mündet. Häufig, so die Beobachtung von Herbert Plügge, verlässt der Kranke dann seinen „Körper nach innen; er zieht sich zurück. In diesem Vorgang verwandelt sich der Leib, indem er immer mehr zurückbleibt zur Hülle“. ${ }^{5}$ Aber es ist eine Hülle, die sich nicht einfach abstoßen lässt und von der man sich auch nur zeitweise distanzieren kann. Kommt ein akuter Schmerzschub, so gelingt nicht nur diese Distanzierung und Entfremdung nicht mehr, im Gegenteil, der Körper ergreift dann die Übermacht über das gesamte Ich in einer unvorstellbaren Radikalität, die Harold Brodkey in seinem Essay Schmerzkontinuum eindrücklich beschrieben hat:

\footnotetext{
${ }^{5}$ Plügge (1962): 69 (Hervorhebung im Text).
} 
Auf einmal und ohne wahrnehmbaren Übergang gleitet man - glitt ich - in eine graue, ölige, schummrig beleuchtete Hülle aus fast totalem Schmerz. Ich könnte sagen, meine missliche Lage nahm meine ganze Aufmerksamkeit dermaßen gefangen, dass mir jegliche vertraute Empfindung entfiel und abhanden kam, dass mir die Unmittelbarkeit der Schmerzlosigkeit abhanden kam. Schmerzlosigkeit schien unendlich weit entfernt. Ich befand mich im Schmerzkontinuum. [...] Noch war ich mir dessen nicht ganz, bewusst - ich war einfach lose eingehüllt [...] in ein irgendwie öliges, schmutziges, halb durchsichtiges, gummiartiges Gefühl der Erschütterung und des Verlustes jeder normalen Verbindung zum Leben. Gewisse Bereiche der körpereigenen Logik setzen - zeitweilig - aus, so etwa die logische Erwartung, ja Gewissheit des nächsten Atemzugs oder der visuellen Wahrnehmung. De facto handelt es sich um den Zusammenbruch eines Großteils der gewöhnlichen Erwartungen, um eine Reihe von Adjustierungen: Schmerz bewirkt höllische Adjustierungen - sie erscheinen ganz und gar endgültig - im Schmerz ist man höllisch weit entfernt vom Licht Gottes. Dort kann man hassen. (Harold Brodkey, Schmerzkontinuum)

Die Erfahrung eines totalen Schmerzes ist die Erfahrung eines kompletten Zusammenbruchs. Die Sinne schwinden, die Wahrnehmung der äußeren Welt löst sich auf, die Hoffnung verdampft, bis zuletzt das Ich überwältigt wird und ebenfalls verschwindet: Ohnmacht.

\section{Die Zeit im Schmerz}

Der Schmerz verändert nicht nur das leibliche Erleben, sondern auch das Erleben der Zeit. Normalerweise wird die Zeit als solche im alltäglichen Leben nicht als etwas Eigenständiges wahrgenommen. „Im Zustand der Normalität verhalten wir uns als kompetente Verwalter unseres Zeitkontos“ ${ }^{6}$, bemerkt Jean-Pierre Wils. Und tatsächlich: Wir planen, nutzen und investieren unsere Zeit. Wir beziehen uns auf die Zukunft durch Pläne, Wünsche und Hoffnungen, die Vergangenheit bleibt in Form von Erinnerungen zurück. Die Gegenwart ist unbeständig: Entweder ist sie noch nicht da, oder sie ist schon nicht mehr. Am ehesten erfahren wir die Zeit als Zeitfluss, als eine Bewegung, die wir nicht explizit wahrnehmen, da sie in einem unmittelbaren Verhältnis zu unserem Leben bzw. zu dem, was wir erleben, steht. Wir Menschen beziehen uns auf das Erlebte, auf die Arbeit, auf Begegnungen, auf Gedanken, auf das Erhoffte oder das Erinnerte, nicht aber auf die Zeit. Dieses „Nicht-Erleben“ der Zeit ändert sich massiv durch den Schmerz, vor allem durch den chronischen, nicht vergehenden Schmerz. „[...] in der Schmerzerfahrung bleibt uns der Zugang zu diesem Zeitkonto verwehrt. Es ist bis auf weiteres gesperrt. ${ }^{67}$

\footnotetext{
${ }^{6}$ Wils (2007): 85.

${ }^{7}$ Ebd. 85.
} 
In zeitlicher Hinsicht bewirkt der Schmerz zunächst einmal eine plötzliche Unterbrechung. Für einen Augenblick wird ein Mensch herausgerissen aus dem Ablauf des Alltags. Der Schmerz, erst recht wenn er akut ist, lässt ihn gebannt an einem Punkt verharren, von dem er nicht zu entkommen vermag. Im Schmerz ist die gesamte Aufmerksamkeit auf die schmerzende Partie des Körpers gerichtet, diese tritt hervor und zwingt den Betroffenen zu einer permanenten Auseinandersetzung mit ihr. Je intensiver und anhaltender der Schmerz ist, desto unausweichlicher die Konfrontation. Dabei gehen sowohl der Ausblick in die Zukunft als auch der Rückblick in die Vergangenheit verloren. Der Betroffene ist im Schmerz gefangen und damit in einer negativen Gegenwart, die nicht vergeht. ${ }^{8}$

Doch was geschieht, wenn der plötzlich eingetretene akute Schmerz andauert, statt zu vergehen? Was geschieht, wenn er chronifiziert? Das Wort „chronisch“ geht zurück auf das Griechische chronos, die „Zeit“. Medizinisch wird der „chronische Schmerz“ in Bezug auf die Dauer seines Bestehens bestimmt. Es ist aber auch möglich, die Chronizität in Bezug auf das Erleben des Schmerzes zu deuten: Der chronische Schmerz ist nicht nur ein Schmerz, der etwas länger dauert. Er ist gerade aufgrund der Zeitkomponente etwas völlig anderes als ein akuter Schmerz, nämlich ein komplexes Leiderlebnis.

Der Schmerz hält zwar in der Gegenwart fest, weist aber zugleich auf die Zukunft hin, denn jeder Schmerz wird per definitionem als ein nicht-sein-sollender empfunden, was bedeutet, dass er die Negation seiner selbst und den Verweis auf das Andere seiner Selbst in sich trägt. Auf zeitlicher Ebene zeigt sich der Bezug auf das Andere durch die Sehnsucht, durch das gespannte Herbeisehnen einer schmerzfreien Zukunft. Im chronischen Schmerz ist der Impuls des ,vergehe!', die Forderung nach sofortiger Beendigung des Schmerzes aber schlichtweg sinnlos: er vergeht nicht und wird auch nie vergehen. ${ }^{9}$ Die Forderung nach der Erlösung von Schmerzen, die der Schmerzerfahrung als solcher inhärent ist, wird im chronischen Schmerz ad absurdum geführt. Während bei einem kurzen Schmerz der Gedanke an eine schmerzfreie Zukunft gerechtfertigt ist und dem Leidenden eine Perspektive und Hoffnung gibt, ist der chronisch Schmerzleidende lebenslänglich dazu verurteilt, in einer Gegenwart zu verharren, aus der heraus keine leidfreie Zukunft möglich ist. Die Zukunft des chronisch Leidenden ist versperrt. Er braucht sich nicht weiter in Geduld zu üben und abzuwarten, dass der Schmerz endlich vergehen möge. Dass er mit dem Warten aufhören muss, ist im Gegenteil eine der wesentlichen Einsichten, zu denen er schließlich gelangen muss. Gelingt ihm dies, so muss er einen Weg finden, sich das Unerträgliche soweit erträglich zu gestalten, dass er es in sein Leben einbinden kann.

\footnotetext{
${ }^{8} \mathrm{Vgl}$. Fuchs (2002).

${ }^{9}$ Vgl. Fuchs (2002): $35 f$.
} 
Der Betroffene muss das Nicht-sein-Sollende in seiner Wirklichkeit akzeptieren, obwohl ihm seine Erinnerung an die verlorene schmerzfreie Vergangenheit immer wieder die Möglichkeit einer schmerzfreien Existenz vorhält. Der Schrei des ,vergehe!‘ muss unterdrückt oder ignoriert werden, um möglichst am Schmerz vorbei leben zu können und so noch ein Stück Freiheit zu bewahren. Die Alternative ist, am Schmerz zu verzweifeln und an ihm unterzugehen.

„Der heftige Schmerz, welcher das Schreien auspresset, lässt entweder bald nach, oder zerstöret das leidende Subjekt. “ (Georg Lessing, Laokoon)

Besonders tückisch ist bei chronischen Schmerzen die Tatsache, dass sie in ihrer Intensität variieren. Es kann Unterbrechungen geben, Phasen, in denen der Schmerz abnimmt, doch wann er abklingen und vor allem, wann er wieder zunehmen wird, ist für den Patienten meistens ungewiss, so dass jeglicher Versuch, ein möglichst strukturiertes Leben jenseits des Schmerzes zu führen, immer wieder aufs Neue zum Scheitern verurteilt ist.

\section{Patientin Monika Roth}

Also, ich kann nicht so drauflos wirtschaften oder wurschteln oder sagen: „, Mensch, morgen machen wir das und dann machen wir das. "Oder mich treffen, mit anderen Leuten. Schon spontan, also eher noch spontan, weil ich dann sagen kann: Jetzt kann ich diese Wanderung gerade mitmachen oder ich könnte jetzt auch anderthalb Stunden laufen. Oder: Jetzt habe ich gerade so Schmerzen mit den Knien und mit der Hüfte und mit dem Rücken, dass ich also nicht so viel laufe...

Die Zeit wird im Schmerz in einer pervertierten Form erfahren, nämlich als nicht vergehende Zeit. Das Drängen nach Heilung, die natürliche Gerichtetheit des Menschen auf die Zukunft hin, ist im Leiden gebunden, gefesselt, so dass ein Sich-Erstrecken in die Zukunft, durch Pläne, Wünsche und Hoffnungen, nicht möglich ist. „Der Leidende wird“, so schildert es Boris Wandruszka, ,,von etwas getroffen, was ihn bindet und an seiner freien und natürlichen Weiterentfaltung [...] hindert: Wie eine Eisenkugel hängt ein Widerfahrnis an ihm, das nicht vergehen will, sondern weiter seine Gegenwart und Zukunft in Beschlag nimmt.“10

Zusammenfassend kann festgehalten werden: Während wir normalerweise die eigene Leiblichkeit und Zeitlichkeit im Modus der Verborgenheit erleben - Leib und Zeit als Medien für den eigenen Lebensvollzug nutzen, ohne sie eigens zum Gegenstand der Wahrnehmung zu machen -, treten sie im Schmerz aus dieser Verborgenheit heraus. Der Schmerz verursacht einen Bruch

\footnotetext{
${ }^{10}$ Wandruszka (2009): 150.
} 
und reißt den Leidenden aus seiner Leib- und Zeitvergessenheit heraus; er zerreißt dadurch gewohnte Zusammenhänge und durchbricht die Kontinuität von Lebensabläufen.

\section{Der Schmerz, das Ich und die Anderen}

Der Schmerz, vor allem der chronische, ist eine totalisierende Erfahrung. ${ }^{11}$ Je nach Intensität und Dauer kann er zu einem mehr oder weniger drastischen Zusammenbruch zwischenmenschlicher Beziehungen und zur völligen Isolation des Leidenden führen. Der Schmerz kann zwischen der eigenen Realitätswahrnehmung und der Realität der Anderen eine unüberwindbare Mauer errichten, was sich nicht zuletzt anhand der Sprache zeigt.

Im Falle akuter Schmerzen ist der Schmerzleidende oftmals nicht einmal mehr in der Lage, seinen Schmerz zu artikulieren, außer vielleicht durch unkontrollierte, spontane Äußerungen wie einen Schrei oder einen verzerrten Gesichtsausdruck. Im Schmerzensschrei ist, wie Elaine Scarry bemerkt, das kommunikative Element derart auf ein Minimum reduziert, dass es für Andere schwer ist, eine angemessene Antwort darauf zu geben. ${ }^{12}$

Im Fall chronischer Schmerzen ist der Schmerz durch seine kontinuierliche Präsenz allgegenwärtig und wird für viele chronische Schmerzpatienten zum einzigen Lebensinhalt.

\section{$\underline{\text { Patientin Petra Andresen }}$}

Es beginnt ja damit, dass Sie vorrangig über Ihre Schmerzen sprechen. Sie merken, Sie haben nichts anderes mehr im Kopf. Dann kommt es, dass Sie sich zurückziehen, weil Sie nicht mehr viel machen können. Sie machen Ihre Arbeit. Das Wochenende findet im Bett statt. Es folgt wieder zunehmende Inaktivität, die natürlich ganz klar auch zu muskulärem Schwund führt und eigentlich ist das dann ein klassischer Werdegang, dass ein Schmerzpatient sich eben total zurückzieht.

Die Leidenden müssen über ihren Schmerz reden können, denn es ist für sie die einzige Möglichkeit einer Objektivierung und dadurch einer (gleichzeitigen) Distanzierung von ihren Schmerzen. Es ist leicht nachvollziehbar, dass dabei die Geduld und das Verständnis der Anderen - oftmals auch der behandelnden Ärzte - an ihre Grenzen kommen, da sie sich keine Vorstellung davon machen können, was es bedeutet, einem kontinuierlichen Schmerz ausgesetzt zu sein. Oft sind nicht die Schmerzen als solche das Schlimmste, sondern die Isolation in der sie die Betroffenen führen.

\footnotetext{
${ }^{11}$ Vgl. Grüny (2004): 34f.

${ }^{12}$ Scarry (1992): 12. Vgl. dazu auch Dietrich (2009).
} 


\section{Patientin Petra Andresen}

Und - also das ist schon, also wie das soziale - das ganze Umfeld, im Arbeitsfeld und auch privat sich total verändert. Also Schmerzen, ein chronischer Schmerzpatient muss schon sehr auf sich aufpassen, weil da droht eine ganz große Isolation.

Und es ist einfach so, dass sich das Umfeld auch schützt. Der Schmerzpatient äußert sich. Und ich bin auch ein Mensch, der nicht nur immer das Schöne, sondern ich sage, wie es mir geht und ich schildere das auch in allen Farben. Und das belastet den Anderen zu sehr. Das können die wenigsten aushalten.

Der Schmerzgeplagte kann keinen normalen Lebensrhythmus mehr einhalten. Er muss seinen Beruf, seine Freizeitaktivitäten aufgeben. Er kann Erwartungen nicht mehr erfüllen. Er kann den verschiedenen Rollen, die jemand im privatem sowie im öffentlichen Leben - als Mitarbeiter, als Mutter, als Partner, als Beraterin usw. - einnimmt, nicht mehr oder nur noch in einer stark reduzierten Weise erfüllen. Das führt zu Rollenverschiebungen, unter Umständen zur Neustrukturierung des gesamten Familien- und Privatlebens, und zur Umverteilung von Aufgaben. Der Schmerzpatient muss sein Leben völlig neu erfinden. Er muss ein Leben mit und um den Schmerz herum gestalten. Dabei ist er stark abhängig von der Hilfe seiner Nächsten und von deren Bereitschaft, ihr Leben ebenfalls neu zu orientieren.

\section{$\underline{\text { Patientin Anja Kaiser }}$}

Es gab sehr viele Streitereien, was ganz schlimm ist und das ist, was sicherlich jeder schon erlebt hat, dieses Garten, Blumen gießen, dann nimmst halt eine halbe Gießkanne, aber wenn dann kommt: „Um Gottes Willen, lass das stehen, du sollst ja nicht lupfen (heben) mit deinem Kreuz" und deine Mutter hat einen Bruch und ist 40 Jahre am Schreibtisch, wirbelsäulengeschädigt und du denkst dann: wenn meine Mutter mal pensioniert ist, entlaste ich sie, ha dann kommt die und sagt: „, Lass das mal stehen, das mach ich. Du mit deinem Kreuz. “, da kommst Du dir vor wie ein unfähiger Krüppel. Du kommst dir unnütz vor in der Familie, abartig und dieses - ich bin ja zu nichts mehr zu gebrauchen, das ist eine ganz gefährliche Einstellung, weil man glaubt sie.

Die Anderen können wichtige Begleiter im Schmerz sein. Sie können aber auch, mit Sartre gesprochen die Hölle sein: Gut gemeinte Ratschläge prasseln auf den Schmerzleidenden nieder und setzen ihn unter Druck. Die gut gemeinte Sorge des Anderen kann leicht in Überfürsorglichkeit und zur Entmündigung des Schmerzleidenden führen. Viele chronische Schmerzpatienten müssen Ihren Beruf aufgeben und geraten dadurch auch noch in finanzielle Abhängigkeit. Auf Ämtern und vor Gutachtern muss ein Betroffener seine Schmerzen, die eigene physische und psychische Verfassung, die eigene Lebensgeschichte und die eigenen Lebensgewohnheiten offenlegen und darauf hoffen, dass er ernst genommen wird, dass er einen Anspruch auf 
staatliche Hilfe, auf Rente bekommt. Er muss sich dem Zweifel, wenn nicht gar dem Misstrauen völlig fremder Menschen aussetzen: Er könnte ja seine Schmerzen auch bloß simulieren. Vielleicht ist er ja nur zu faul um zu arbeiten, vielleicht ist der Schmerz - den Andere ja nie direkt spüren oder sehen können - nur eingebildet. Vielleicht müsste er doch einfach mal die Zähne zusammenbeißen, andere hätten es schließlich auch nicht leicht... Der Zweifel, immer noch nicht genug gegen den Schmerz unternommen zu haben, gar versagt zu haben, nistet sich auch bei dem Betroffenen selbst ein. Das Selbstvertrauen bröckelt.

Durch den Verlust von Aufgaben, dem erzwungenen Rückzug aus der Arbeit und aus sonstigen Aktivitäten machen sich bei vielen chronischen Schmerzleidenden ein Gefühl von Nutzlosigkeit breit und die Angst, den Anderen nur noch zur Last zu fallen.

\section{$\underline{\text { Patientin Daniela Klein }}$}

Das ist dann schon schlimm, wenn du dann so denkst: Menschenskinder, so alt bist du gar nicht und auf einmal hat die Welt keine Verwendung mehr für dich. Und ich weiß dann echt nicht, was ich machen soll. Ich weiß es wirklich nicht. Mir fehlt mein Platz in dieser Welt.

Währenddessen nimmt das Leben der Anderen den gewohnten Lauf: Sie erfüllen ihre Aufgaben, ihre Pläne und Wünsche, genießen das Leben und wollen dabei nicht gestört werden. Vor allem in Gesellschaften, in denen alles immer schneller, einfacher und besser gehen soll, die auf Funktionalität und Leistungsfähigkeit ausgerichtet sind, macht sich das Gefälle zwischen sozialen Erwartungen und dem, was ein chronisch Schmerzleidender leisten kann, für die Betroffenen schmerzlich bemerkbar.

\section{Patientin Anna Wagner}

Ein Aspekt [...] das trifft wahrscheinlich zu für jede Art von chronischer Krankheit - so doch die Erfahrung, dass Gesunde und Kranke doch in verschiedenen Welten leben. [...] Und das ist auch niemandes Schuld oder das will auch niemand so, das hängt in der Natur der Situation einfach. - [...] Weil bestimmte Erfahrungen kann man so auch gar nicht teilen dass, kann man auch nicht erwarten, weil ja einfach der gesunde Mensch in einem völlig anderen Rhythmus auch lebt dann. Und eben der chronisch Kranke oder der - jetzt in dem Fall der chronisch Schmerzkranke - er stirbt nicht, er wird aber auch nicht gesund. [...] - Und das hört nicht auf....

Die Schmerzpatienten sind die Zurückbleibenden. Die, welche auch andere zurückhalten. Sie fallen aus sozialen Beziehungen und Arbeitsverhältnissen heraus. Aktivitäten und Beziehungen, die bisher das Leben ausgemacht hatten, fallen weg. Die Isolation, die Frustration, das Gefühl von Verlorenheit in einer Welt, in der sie keine Aufgabe mehr erfüllen, keine Bedeutung mehr zu haben scheinen und in der sich Hoffnungslosigkeit breit macht, die daraus erwächst, 
dass dieser Zustand sich nie verändern wird, treiben sie nicht selten an den Abgrund der Verzweiflung. Der chronische Schmerzpatient „stirbt noch nicht mal“" Es ist kein Ende, keine Erlösung in Sicht.

\section{Der Schmerz, das Ich und die eigene Existenz}

Solange man gesund ist, existiert man nicht. Genauer gesagt: man weiß nicht, dass man existiert. (Emil Cioran)

Der Schmerz lässt die leibliche und zeitliche Verfasstheit menschlichen Lebens zum Vorschein kommen. Er schneidet den Leidenden von seinem gewohnten Leben ab, hält ihn von den Anderen fern, zwingt ihn zur Isolation. Gefangen in seiner Einsamkeit und Verzweiflung kann ein leidender Mensch kaum umhin, sich der Frage nach dem Warum zu stellen.

\section{Patientin Christa Schumacher}

Und dann kommen eben noch die Gedanken dazu: Warum eigentlich? Warum habe ich das alles? Das ist manchmal gar nicht so einfach, das zu verstehen. Denn es ist ja nicht nur diese eine Geschichte. Ich bin ja schon in ganz frühen Jahren eben auch durch andere Krankheiten gebeutelt gewesen und das kann man dann irgendwann auch nicht mehr ertragen und fragt dann: Was habe ich bloß verbrochen, dass man so leben muss, wie ich lebe? Ich würde auch gerne noch auf die grüne Wiese gehen.

Die Frage nach dem Warum drängt sich unweigerlich auf., denn Menschen orientieren sich gewöhnlich an plausiblen Erklärungen und bedürfen stimmiger Sinndeutungen. Der chronische, nicht enden wollende Schmerz, wie andere Leiderfahrungen auch sprengt jedoch jede Form von Erklärung und Sinngebung. Medizinisch gesehen erfüllt er in den meisten Fällen keine Funktion - anders als der akute Schmerz, der immerhin eine Warnfunktion hat. Er schützt auch das Leben nicht, er zehrt es vielmehr auf. Auch psychosomatisch bedingte oder erklärte Schmerzen sind nicht immer funktional, auch wenn ihnen allzu gerne eine Bedeutung zugeschrieben wird. Und so hinterfragt ein betroffener Mensch sich pausenlos, analysiert akribisch die eigene Biographie, das eigene Verhalten, die Umgebung. Mit Hilfe eines Therapeuten geht er auf die Suche nach jenem Trauma, jenem Fehlverhalten, das als Ursache für den Schmerz dienen könnte. Bei einigen Menschen kann tatsächlich ein traumatisches Erlebnis als Ursprung des Schmerzes ausgemacht werden. Doch ist das eine sinnstiftende Erklärung? Bei anderen lässt sich natürlich auch etwas finden: Jemand sei in der Vergangenheit nicht achtsam zu sich 
selbst gewesen, habe nicht auf sich gehört. In diesen und ähnlichen Erklärungsversuchen verbirgt sich der verzweifelte Versuch, die Kontrolle zu bewahren, und dies - wohl bemerkt sowohl von Seiten des Patienten als auch von Seiten der Ärzte und Therapeuten. Wenn nicht davon ausgegangen werden kann, dass der Schmerz erklärt und somit kontrolliert werden kann, macht auch die Arbeit daran keinen Sinn mehr. Doch diese Situation ist durchaus zweischneidig: dem Einen oder Anderen ist es sicherlich eine Hilfe, sich auf eine Veränderung des eigenen Verhaltens und der eigenen Lebensweise zu konzentrieren. Vielleicht bietet diese Haltung sogar eine Ablenkung vom Schmerz. Für Andere kann sich der Versuch, Erklärungen zu finden, fatal auswirken. Das ist insbesondere dann der Fall, wenn jemand zur Überzeugung gelangt, er habe tatsächlich etwas falsch gemacht und dadurch den Schmerz selbst verursacht. Dann geht es um die eigene Verantwortung und letztlich Schuld für die eigene Misere. In den meisten Situationen müsste jedoch schlichtweg akzeptiert werden, dass letztlich keine Schuld in einem angemessenen und nachvollziehbaren Verhältnis zu erlittenen Schmerzen steht. Das wiederum bedeutet, dass die Frage nach dem „Warum?“ oftmals nicht beantwortet werden kann, dass der Schmerz sich nicht nur der Kontrolle, sondern auch einer vordergründigen Sinnantwort entzieht. Verselbständigt sich schließlich der Schmerz und nimmt er - in seiner ganzen Unsinnigkeit und Sinnwidrigkeit - eine zentrale Stelle im Identitäts- und Selbsterleben eines Menschen ein, dann droht die gesamte Existenz sinnlos zu werden. Ein Suizid kann dann als die einzige, plausible Antwort auf den Schmerz scheinen.

Dem anscheinend Sinnwidrigen und Absurden dennoch einen Sinn abzugewinnen ist die erstaunliche Leistung, die Einigen Schmerzleidenden dennoch gelingt. Den Schmerz im Sinne einer Reifung der eigenen Persönlichkeit, als Anstoß zu einer von Demut und Dankbarkeit geprägten Grundhaltung zu erleben und zu deuten, wie es Maria Schmitz beschreibt. Im Sinne einer Hoffnung wider alle Hoffnung soll ihr Zeugnis am Ende dieser kurzen Phänomenologie des Schmerzes stehen:

\section{$\underline{\text { Patientin Maria Schmitz }}$}

Und Sinn des Schmerzes: es lehrt im gewissen Sinne eine Reue, dass man nicht so hochnäsig wird (lacht). Weiß ich nicht, ich betrachte das dabei nicht als Strafe vom lieben Gott, dass er mich bestraft hat, dass das jetzt - warum ich und sonst keiner, sondern ich denke, es mäßigt einen auch. Dass man, dass jeder ein Maß im Leben bekommt, und mit meinem kann ich dann gut umgehen, da möchte ich nicht mit vielen anderen tauschen.

\section{Literaturverzeichnis}

Bozzaro, Claudia, Das Leiden an der verrinnenden Zeit. Eine ethisch-philosophische 
Untersuchung zum Zusammenhang von Alter, Leid und Zeit am Beispiel der Anti-AgingMedizin. Stuttgart- Bad Cannstatt (2014)

Dietrich, Julia, >Ich habe Schmerzen ‘. Anthropologische Grundlagen des Verhältnisses von Schmerz und Sprache. In: Ingensiep, Hans-Werner/Rehbock, Theda (Hrsg.): »Die rechten Worte finden...«. Sprache und Sinn in Grenzsituationen des Lebens. Würzburg (2009), S. 107-122

Fuchs, Thomas, Zeit-Diagnosen. Philosophisch-psychiatrische Essays. Zug (2002)

Grüny, Christian, Zerstörte Erfahrung. Eine Phänomenologie des Schmerzes. Würzburg (2004)

Plügge, Herbert, Wohlbefinden und Missbefinden. Beiträge zu einer medizinischen Anthropologie. Tübingen (1962)

Scarry, Elaine, Der Körper im Schmerz. Die Chiffren der Verletzlichkeit und die Erfindung der Kultur. Frankfurt am Main (1992)

Wandruszka, Boris, Philosophie des Leidens. Zur Seinsstruktur des pathischen Lebens. Freiburg (2009)

Wils, Jean-Pierre, Ars Moriendi: Über das Sterben. Frankfurt am Main (2007)

www.krankheitserfahrungen.de (zuletzt abgerufen am 21.12.2014).

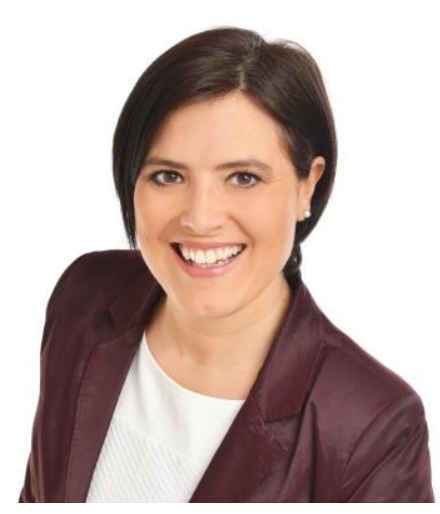

DR. CLAUDIA BOZZARO ist geboren in den USA und aufgewachsen in Italien. Sie studierte Philosophie und Kunstgeschichte in Freiburg und Paris. Ihr Forschungsgebiet befasst sich mit Themen wie Ethik am Lebensende, Ethikberatung, Konzepte von Schmerz und Leiden in der Medizin, philosophische Anthropologie, Existenzphilosophie unter anderen. In 2017 bekam sie ihre dritte Auszeichnung bei der Universität Freiburg: „Albert-Bürklin-Preis der Freiburger Wissenschaftsgesellschaft"“. 